\title{
Эволюция электронного транспорта при резистивных переключениях в пленках порфиразинов
}

\author{
() К.А. Дроздов ${ }^{1}$, И.В. Крылов ${ }^{1}$, В.А. Василик ${ }^{1}$, А.Д. Косов ${ }^{1}$, Т.В. Дубинина ${ }^{2,3}$, \\ Л.И. Рябова ${ }^{2}$, Д.Р. Хохлов ${ }^{1,4}$ \\ ${ }^{1}$ Московский государственный университет им. М.В. Ломоносова (физический фракультет), \\ 119991 Москва, Россия \\ ${ }^{2}$ Московский государственный университет им. М.В. Ломоносова (химический фракультет), \\ 119991 Москва, Россия \\ ${ }^{3}$ Институт физиологически активных веществ Российской академии наук, \\ 142432 Черноголовка, Московская область, Россия \\ ${ }^{4}$ Физический институт им. П.Н. Лебедева Российской академии наук, \\ 119333 Москва, Россия \\ E-mail: kadrozdov@gmail.com
}

Поступила в Редакцию 22 октября 2020 г.

В окончательной редакции 12 ноября 2020 г.

Принята к публикации 12 ноября 2020 г.

\begin{abstract}
Анализ вольт-амперных характеристик позволил определить механизмы проводимости, соответствующие различным состояниям каналов протекания при резистивных переключениях в пленках порфиразинов. Варьирование температуры, структуры диэлектрической матрицы и типа основных носителей заряда позволило оценить применимость модели проводящих каналов для описания транспорта и определить механизмы проводимости для каждого состояния системы.
\end{abstract}

Ключевые слова: пленки порфиразинов, транспорт носителей заряда, резистивное переключение.

DOI: $10.21883 /$ FTP.2021.03.50598.9540

\section{1. Введение}

Разработка быстрых, компактных и дешевых элементов электронной памяти является одной из приоритетных задач современной микроэлектроники [1]. Принципы работы резистивной памяти предполагают возможность системы переключаться между двумя состояниями „Оп“ и „Оff“ с разными сопротивлениями. В настоящее время существует несколько десятков различных подходов к моделированию процесса резистивных переключений [2] с различными экспериментальными данными в пользу каждого из них. Для неорганических материалов механизм резистивных переключений и транспорт носителей заряда в каждом состоянии системы достаточно хорошо изучен $[2,3]$. Однако для органических материалов большая часть этих моделей не применима ввиду физических ограничений или вычислительной сложности, а дополнительные эффекты, характерные для органических материалов, приводят к недостоверности предсказаний оставшейся части [4].

В настоящей работе планируется учитывать несколько различных существующих механизмов для получения корректного представления о резистивных переключениях и проводимости в каждом состоянии системы.

Цель настоящей работы - моделирование состояния системы за счет варьирования параметров (температура, структура органической матрицы, тип основных носителей заряда) и определение механизмов проводимости для каждого состояния системы.

\section{2. Методика эксперимента и исследованные образцы}

В настоящей работе объектами исследования являлись пленки порфиразинов: фенилзамещенного пиразинопорфиразинового комплекса эрбия (PhPorphErOAc), гетеролептического нафталоцианинато-фталоцианината лютеция (PhNcLuPhPc), а также фенилзамещенного субфталоцианина с центральным атомом бора и бромом в качестве аксиального лиганда (PhSubPcBBr) (рис. 1). Выбор данных материалов обусловлен их хорошей растворимостью в органических растворителях, термической и фотохимической устойчивостью и уменьшенной склонностью к агрегации [5,6], а также различием в структурных параметрах и типе основных носителей заряда. PhPorphErOAc является полупроводником $n$-типа [7]. При этом строение пиразинопорфиразинового макрокольца в PhPorphErOAc практически плоское. PhSubPcBBr и PhNcLuPhPc, как и большинство фталоцианинов, являются полупроводниками $p$-типа [8]. Молекула субфталоцианина $\mathrm{PhSubPcBBr}$ имеет конусообразную форму [9]. Лютециевый комплекс PhNcLuPhPc относится к сандвичевому типу молекул, в которых центральный ион металла расположен между двумя органическими лигандами.

Синтез осуществлялся в соответствии с описанными протоколами для PhPorphErOAc [10], PhSubPcBBr [11] и $\mathrm{PhNcLuPhPc}[12]$. Концентрации растворов, подготовленных для нанесения на подложку, составляли PhPorphErOAc $\left(1.6 \cdot 10^{-4} \mathrm{M}\right.$ в смеси бензол:пиридин 


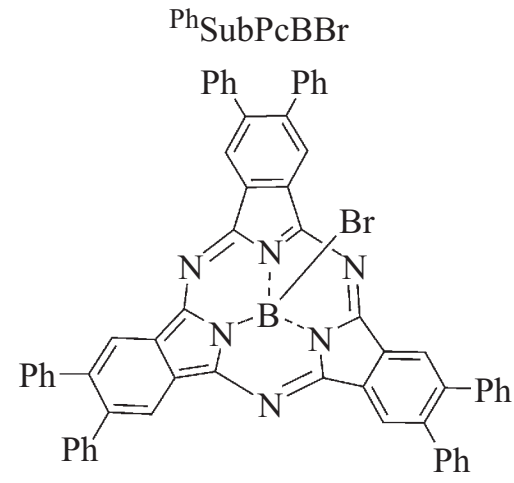

${ }^{\mathrm{Ph}} \mathrm{NcLu}^{\mathrm{Ph} P \mathrm{c}}$

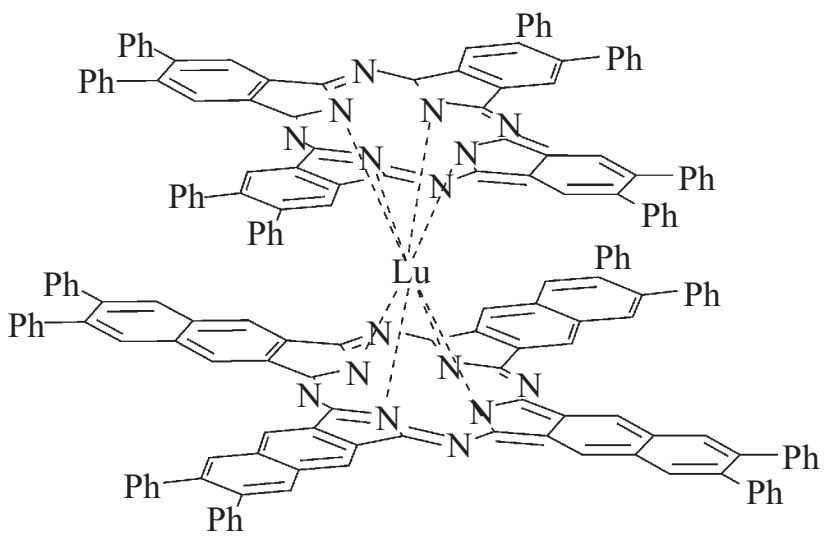

${ }^{\mathrm{Ph}}$ PorphErOAc

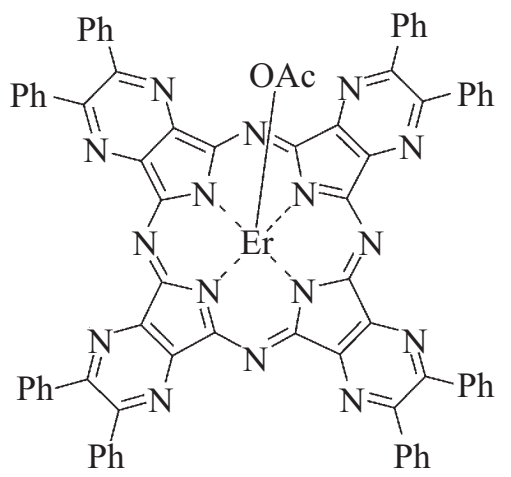

Рис. 1. Схематическое строение макромолекул исследуемых образцов.

$(1: 4, \mathrm{~V}: \mathrm{V})), \mathrm{PhNcLuPhPc}\left(7.6 \cdot 10^{-5} \mathrm{M}\right.$ в бензоле), а также $\mathrm{PhSubPcBBr}\left(2.4 \cdot 10^{-4} \mathrm{M}\right.$ в бензоле).

Образцы представляли собой сандвич-структуры. Раствор активного компонента наносился капельным методом на диэлектрическую подложку с серебряными контактами [13]. Верхний контакт формировался с помощью серебряной пасты „Контактол“. По данным оптической микроскопии толщина активного слоя составляла $\approx 2$ мкм. Изготовление образцов производилось при температуре $300 \mathrm{~K}$ в атмосферных условиях.
Для проведения измерений образец устанавливался в камеру, экранирующую внешнее излучение. Камера вакуумировалась и заполнялась парами гелия.

Измерения вольт-амперных характеристик производились с помощью прибора Keithley 2612A SourceMeter в диапазоне электрических напряжений от 0 до $100 \mathrm{~B}$, скорости развертки от 0.3 до $30.0 \mathrm{MB} / \mathrm{c}$.

Добавление в измерительную схему нагрузочного резистора последовательно с образцом позволяло перераспределить напряжение между образцом и нагрузочным резистором. Варьирование величины нагрузочного сопротивления от 1 Ом до 1.66 МОм позволило изменять соотношение тока и напряжения на образце и, как следствие, зафиксировать образец в проводящем, метастабильном или непроводящем состоянии [14].

\section{3. Результаты измерений}

Эффект резистивного переключения заключается в обратимом переходе структуры между состояниями с различной проводимостью. Для всех пленок наблюдается два устойчивых состояния - проводящее („Оn“) и непроводящее („Off“). Вольт-амперная характеристика проводящего состояния линейна, значения сопротивления составляют 5Ом, 8.2 КОм, 188 КОм для пленок $\mathrm{PhPorphErOAc,} \mathrm{PhSubPcBBr}$, PhNcLuPhPc соответственно. Сопротивление непроводящего состояния превышает 1 ГОм для всех образцов.

Для пленок $\mathrm{PhNcLuPhPc}$ и $\mathrm{PhSubPcBBr}$ между устойчивыми „Оn“ и „Оff“ состояниями можно реализовать серию метастабильных состояний. Типичные вольт-амперные характеристики пленок представлены на рис. 2. Значения напряжения на образце не превышали 15 В. Увеличение напряжений на образце до зна-

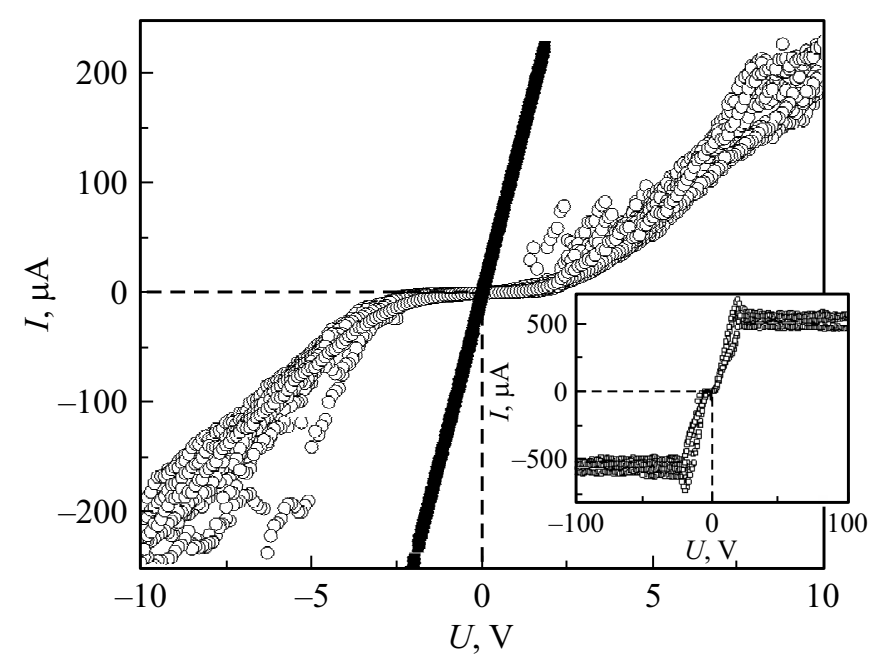

Рис. 2. Вольт-амперные характеристики пленок $\mathrm{PhSubPcBBr}$. Закрытые символы отвечают проводящему, открытые - метастабильным состояниям. $T=300 \mathrm{~K}$. На вставке приведена вольт-амперная характеристика пленок $\mathrm{PhSubPcBBr}$ при напряжении на образце, превышающем 25 В. $T=300 \mathrm{~K}$. 


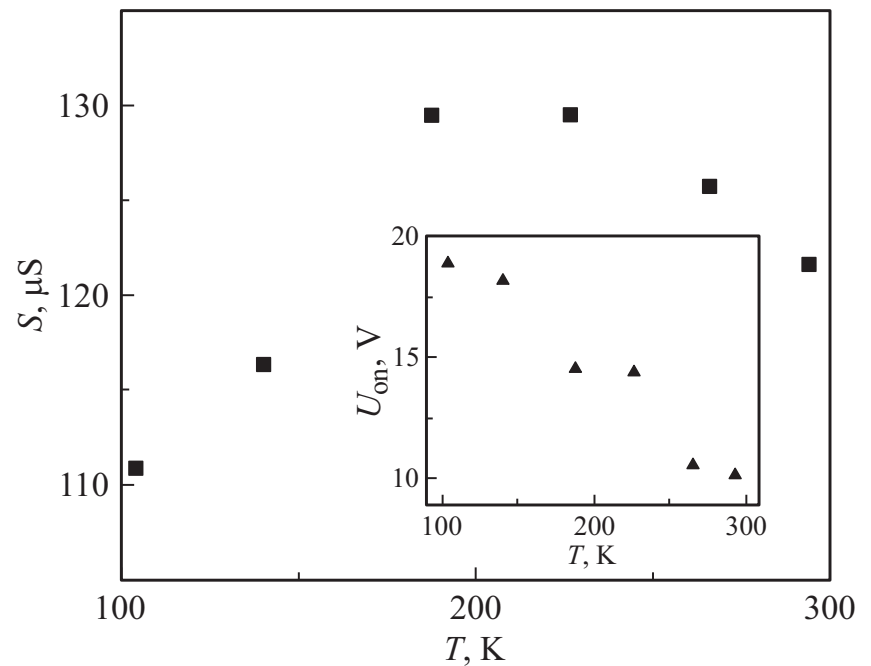

Pис. 3. Температурная зависимость проводимости для пленок $\mathrm{PhSubPcBBr}$. На вставке представлена температурная зависимость напряжений переключений в проводящее состояние $U_{\text {on }}$ для пленок $\mathrm{PhSubPcBBr}$.

чений, превышающих $25 \mathrm{~B}$, приводит к возникновению обратимого состояния стабилизации тока (см. вставку на рис. 2). В режиме стабилизации тока проводящее состояние не наблюдается. Независимо от приложенного к образцу напряжения вольт-амперные характеристики метастабильных состояний нелинейны, но симметричны. Для пленок PhPorphErOAc реализовать метастабильные состояния не удалось, разрушение проводящего состояния происходило при протекании через образец тока $\sim 0.1 \mathrm{~A}$.

Для пленок PhSubPcBBr были проведены измерения вольт-амперных характеристик в диапазоне температур 100-300 К. Для полученных кривых общий вид вольт-амперных характеристик качественно не меняется. Температурная зависимость проводимости пленки в состоянии „Оп“ приведена на рис. 3. Проводимость меняется немонотонно с локальным максимумом вблизи $200 \mathrm{~K}$. На вставке к рис. 3 приведена температурная зависимость напряжений резистивных переключений в проводящее состояние $U_{\text {on }}$. С понижением температуры наблюдается монотонный рост $U_{\text {on. }}$ Все полученные температурные зависимости обратимы при охлаждении и нагреве.

\section{4. Обсуждение результатов}

Согласно данным работ $[1,15]$, проводимость в метастабильном и проводящем состоянии обусловлена наличием в активном слое проводящих каналов, общий объем которых незначителен по сравнению с объемом активного слоя. Так как вольт-амперные характеристики для проводящего состояния всех пленок (рис. 2) линейны, для носителей заряда отсутствует барьер при переходе между контактом и каналом.
Различия в несколько порядков для сопротивления проводящего состояния пленок PhPorphErOAc, $\mathrm{PhSubPcBBr}$ и $\mathrm{PhNcLuPhPc}$ связаны с различным диаметром единичного канала $[15,16]$ и различной плотностью каналов в единице объема [14]. Формирование канала обусловлено диффузией ионов металла из контакта [17]. Большие линейные размеры молекул в пленках $\mathrm{PhNcLuPhPc} \mathrm{[12]} \mathrm{по} \mathrm{сравнению} \mathrm{с} \mathrm{PhPorphErOAc} \mathrm{[18]} \mathrm{и}$ $\mathrm{PhSubPcBBr}[19]$ затрудняют диффузию. Отрицательно заряженные молекулярные остовы в пленках $p$-типа $\mathrm{PhNcLuPhPc}$ и $\mathrm{PhSubPcBBr}$ препятствуют формированию канала из диффундировавших в пленку ионов $\mathrm{Ag}^{+}$.

Согласно данным работ $[15,16]$, значения напряжений переключений в проводящее состояние $U_{\text {оn }}$ (см. вставку на рис. 2) задаются выражением $U_{\text {on }}=F_{c}\left(L+D\left(\rho_{d} / \rho_{f}-1\right)\right)$, где $F_{c}-$ напряжение приложенного поля, $L$ - толщина активного слоя, $D-$ ширина барьера, $\rho_{d}$ и $\rho_{f}-$ удельные сопротивления диэлектрической матрицы и канала соответственно. Для канала с металлическим типом проводимости и диэлектрической матрицы соотношение $\rho_{d}$ и $\rho_{f}$ будет возрастать с понижением температуры, что объясняет наблюдаемый рост $U_{\text {on }}$ на рис. 3 .

Проводимость по каналам является металлической, процесс формирования каналов зависит от температуры образца. Понижение температуры сопровождается уменьшением толщины каналов [15,20], что приводит к росту сопротивления образца. Эти факторы обусловливают немонотонную зависимость проводимости от температуры на рис. 3 .

Проводимость в метастабильном состоянии определяется механизмами транспорта носителей заряда через барьеры, разделяющие фрагменты каналов. Для пленок $\mathrm{PhNcLuPhPc}$ транспорт носителей заряда через барьеры осуществляется по механизму Френкеля-Пула [21] (рис. 4) начиная со значений напряжений $U>1 \mathrm{~B}$. Зависимость проводимости $\sigma$ от напряженности прило-

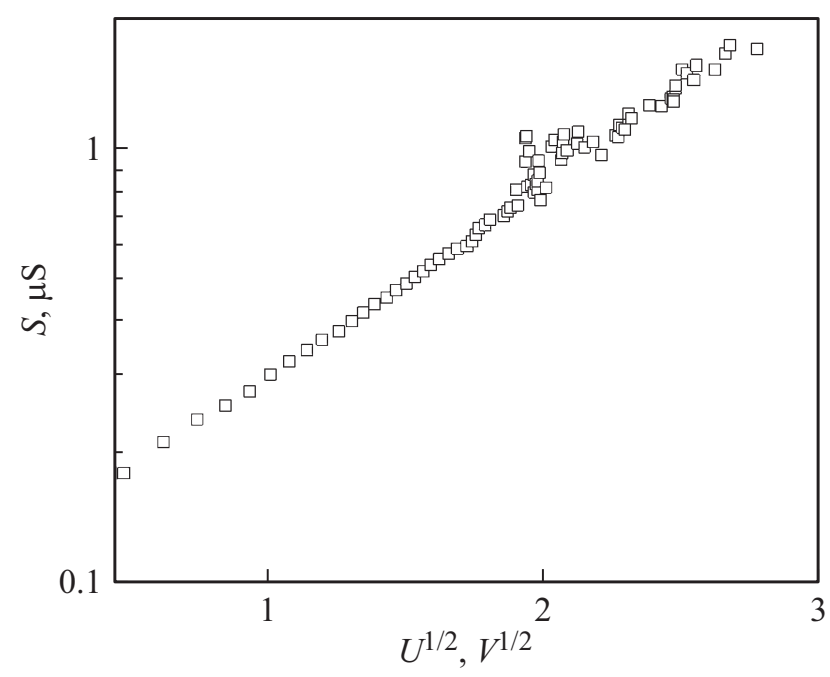

Рис. 4. Проводимость метастабильного состояния пленки PhNcLuPhPc. 


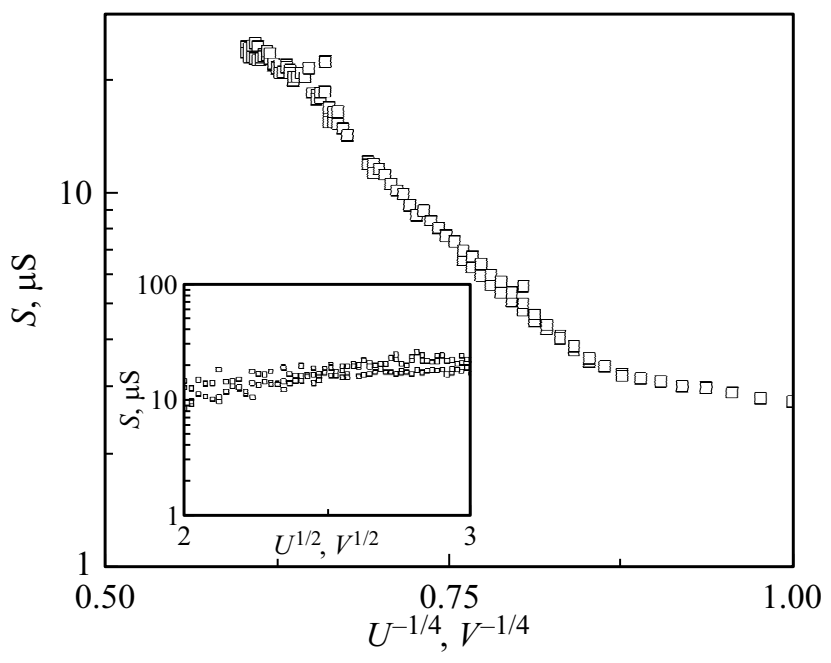

Рис. 5. Проводимость метастабильного состояния пленки $\mathrm{PhSubPcBBr} \mathrm{по} \mathrm{прыжковому} \mathrm{механизму} \mathrm{в} \mathrm{сильных} \mathrm{полях.} \mathrm{На}$ вставке приведена проводимость метастабильного состояния пленки PhSubPcBBr по механизму Френкеля-Пула.

женного поля $E$ для механизма Френкеля-Пула задается соотношением $\sigma=A_{1} \exp \left(B_{1} E^{1 / 2}\right)$.

В малых полях вольт-амперные характеристики метастабильных состояний близки к линейным. Из их вида по модели Пула $[21,22]$ можно оценить среднее расстояние между барьерами $\langle a\rangle$. Выражение $2 k T \approx e\langle a\rangle E$ дает величину $\langle a\rangle \approx 0.1-0.2$ мкм.

Для пленок $\mathrm{PhSubPcBBr}$ транспорт носителей заряда через барьеры осуществляется по прыжковому механизму в сильных полях [23] (рис. 5) при напряжении $0.5<U<4$ В и механизму Френкеля-Пула (см. вставку на рис. 5) при напряжении $U>4$ В. Зависимость проводимости $\sigma$ от напряженности приложенного поля $E$ для прыжкового механизма в сильном поле задается соотношением $\sigma=A_{2} \exp \left(-B_{2} E^{-1 / 4}\right)$.

Оценки среднего расстояния между барьерами из границ линейности вольт-амперных характеристик для различных метастабильных состояний дают величину $\langle a\rangle \approx 1.0-0.2$ мкм.

Таким образом, из оценок характерных расстояний между барьерами можно утверждать, что в метастабильном состоянии в пленках $\mathrm{PhNcLuPhP}$ перенос электрона между контактами сопровождается прохождением от 10 до 20 барьеров, в пленках $\mathrm{PhSubPcBBr}$ - от 2 до 10. Уменьшение сопротивления метастабильного состояния сопровождается уменьшением среднего числа барьеров в активном слое. Данный результат находится в хорошем соответствии с данными [14], где было показано, что переход в более низкоомные состояния сопровождается процессом формирования перколяционной сети, в которой транспорт может осуществляться в обход участка с локальным разрывом канала. Активационный механизм Френкеля-Пула доминирует для структур с небольшим числом барьеров, где на каждом барьере падает существенное напряжение. Увеличение числа барьеров в структурах смещает область активационной проводимости Френкеля-Пула в сторону больших напряжений.

Выход вольт-амперных кривых на режим стабилизации тока для пленок $\mathrm{PhSubPcBBr}$ и PhNcLuPhPc (cм. вставку на рис. 2) обусловлен увеличением ширины барьера при разрыве канала на более высоких значениях напряжения $[16,24]$ вследствие больших термических флуктуаций.

\section{5. Заключение}

Исследованы вольт-амперные характеристики пленок PhPorphErOAc, PhSubPcBBr, PhNcLuPhPc при комнатной температуре, эволюция вольт-амперных характеристик при изменении температуры и варьировании соотношения тока и напряжения за счет различных нагрузочных сопротивлений.

Во всех структурах наблюдаются резистивные переключения в проводящее состояние при значениях напряжения, превышающих критические. Транспорт носителей заряда в проводящем состоянии осуществляется по каналам, формирующимся вследствие диффузии атомов металла из контакта. Процесс диффузии и формирования каналов зависит от свойств среды. Толщина каналов и их плотность в единице объема определяют значения проводимости. Наибольшие значения проводимости получены для пленок $n$-типа с макромолекулами малых размеров и планарной геометрией (PhPorphErOAc), наименьшие - для пленок $p$-типа с макромолекулами наибольшего размера и бипланарной геометрией $(\mathrm{PhNcLuPhPc})$. Показано, что понижение температуры приводит к уменьшению толщины канала и уменьшению проводимости.

Разрушение проводящего канала происходит из-за термических флуктуаций вследствие локального разогрева. Большая толщина каналов и большая плотность каналов в единице объема (PhPorphErOAc) позволяют получить проводящее состояние, устойчивое к разрушению.

Метастабильные состояния в пленках $\mathrm{PhSubPcBBr}$ и $\mathrm{PhNcLuPhPc}$ отвечают ситуации, когда сеть каналов в структуре представлена отдельными фрагментами, разделенными энергетическими барьерами. Доминирующие механизмы транспорта определяются соотношением числа барьеров и величины приложенного к структуре напряжения. Малым значениям напряжения и большому числу барьеров отвечает прыжковая проводимость. Увеличение напряжения увеличивает вклад активационной проводимости Френкеля-Пула.

Таким образом, показано, что характер транспорта носителей заряда для всех рассмотренных пленок PhPorphErOAc, PhSubPcBBr, PhNcLuPhPc с различным типом основных носителей заряда и различными линейными размерами и структурами макромолекул может быть описан в рамках модели проводящих каналов. 


\section{Финансирование работы}

Исследование выполнено при финансовой поддержке РФФИ в рамках научного проекта № 20-32-70118.

\section{Конфликт интересов}

Авторы заявляют, что у них нет конфликта интересов.

\section{Список литературы}

[1] F. Pan, S. Gao, C. Chen, C. Song, F. Zeng. Mater. Sci. Eng., R, 83, 1 (2014).

[2] D. Panda, P.P. Sahu, T.Yu. Tseng. Nanoscale Res. Lett., 13 (1), Art. \#8 (2018).

[3] В.К. Ярмаркин, С.Г. Шульман, В.В. Леманов. ФТТ, 50 (10), 1767 (2008).

[4] J.C. Scott, L.-D. Bozano. Adv. Mater., 19 (11), 1452 (2007).

[5] Y. Chen, D. Li, N. Yuan, J. Gao, R. Gu, G. Lu, M. Bouvet. J. Mater. Chem., 22, 22142 (2012).

[6] T.V. Dubinina, K.V. Paramonova, St.A. Trashin, N.E. Borisova, L.G. Tomilova, N.S. Zefirov. Dalton Trans., 43, 2799 (2014).

[7] D. Schlettwein, D. Woehrle, E. Karmann, U. Melville. Chem. Mater., 6 (1), 3 (1994).

[8] R.D. Gould. Coord. Chem. Rev., 156, 237 (1996).

[9] Ch.G. Claessens. Chem. Rev., 114 (4), 2192 (2014).

[10] A.D. Kosov, T.V. Dubinina, N.E. Borisova, A.V. Ivanov, K.A. Drozdov, S.A. Trashin, K. De Wael, M.S. Kotova, L.G. Tomilova. New J. Chem., 43, 3153 (2019).

[11] T.V. Dubinina, M.M. Osipova, A.V. Zasedatelev, V.I. Krasovskii, N.E. Borisova, S.A. Trashin, L.G. Tomilova, N.S. Zefirov. Dyes and Pigments, 128, 141 (2016).

[12] T.V. Dubinina, A.D. Kosov, E.F. Petrusevich, S.S. Maklakov, N.E. Borisova, L.G. Tomilova, N.S. Zefirov. Dalton Trans., 44, 7973 (2015).

[13] М.С. Котова, М.А. Дронов, Т.В. Дубинина, Д.Р. Хохлов. Физ. образование в вузах, 21 (1C), 53 (2015).

[14] M.S. Kotova, K.A. Drozdov, T.V. Dubinina, E.A. Kuzmina, L.G. Tomilova, R.B. Vasiliev, A.O. Dudnik, L.I. Ryabova, D.R. Khokhlov. Sci. Rep., 8, 9080, 1 (2018).

[15] D. Ielmini. Semicond. Sci. Technol., 31, 063002 (2016).

[16] D. Ielmini. IEEE Trans. Electron Dev., 58, 4309 (2011).

[17] I. Valov, R. Waser, J.R. Jameson, M.N. Kozicki. Nanotechnology, 22, 254003 (2011).

[18] M.P. Donzello, C. Ercolani, V. Novakova, P. Zimcik, P.A. Stuzhin. Coord. Chem. Rev., 309, 107 (2016).

[19] C.G. Claessens, D. González-Rodríguez, M.S. Rodríguez-Morgade, A. Medina, T. Torres. Chem. Rev., 114 (4), 2192 (2014).

[20] S. Ambrogio, S. Balatti, S. Choi, D. Ielmini. Adv. Mater., 26, 3885 (2014).

[21] Б.И. Шкловский. ФТП, 13 (1), 93 (1979).

[22] Р.Б. Васильев, М.Н. Румянцева, Л.И. Рябова, А.М. Гаськов. ФТП, 43 (2), 167 (2009).

[23] Н. Мотт, Э. Дэвис. Электронные процессы в некристаллических веществах (М., Мир, 1992) т. 1, с. 47.

[24] U. Russo, D. Ielmini, C. Cagli, A.L. Lacaita. IEEE Trans. Electron Dev., 56 (2), 193 (2009).

\section{Evolution of electron transport under resistive switching in porphyrazine films}

K.A. Drozdov ${ }^{1}$, I.V. Krylov' ${ }^{1}$, V.A. Vasilik ${ }^{1}$, A.D. Kosov' ${ }^{2}$, T.V. Dubinina ${ }^{2,3}$, L.I. Ryabova ${ }^{2}$, D.R. Khokhlov ${ }^{\mathbf{1 , 4}}$

${ }^{1}$ Lomonosov Moscow State University

(Department of Physics), 119991 Moscow, Russia

${ }^{2}$ Lomonosov Moscow State University

(Department of Chemistry),

119991 Moscow, Russia

${ }^{3}$ Institute of Physiologically Active Compounds,

Russian Academy of Sciences,

142432 Chernogolovka, Moscow Region, Russia

${ }^{4}$ Lebedev Physical Institute,

Russian Academy of Sciences,

119333 Moscow, Russia

Abstract Analysis of the current-voltage characteristics allowed to determine the conductivity mechanisms, corresponding to different states of the conducting filaments during resistive switching in porphyrazine films. Varying the temperature, the structure of the dielectric matrix, and the type of the main charge carriers made it possible to assess the applicability of the model of conducting filaments to describe transport and to determine the mechanisms of conductivity for each state of the system. 